\title{
Prospective helical acquisition for coronary CT angiography
}

\author{
Marc Dewey
}

Received: 29 October 2009/Accepted: 30 October 2009/Published online: 18 November 2009

(C) Springer Science+Business Media, B.V. 2009

\begin{abstract}
Abbreviations
CAD Coronary artery disease

CT Computed tomography

ECG Electrocardiogram
\end{abstract}

Noninvasive detection of coronary artery disease (CAD) using computed tomography (CT) with multiple detector rows has high sensitivity and negative predictive value $[1,2]$ and is thus considered an appropriate clinical option to reliably rule out CAD in patients with low-to-intermediate pretest likelihood of disease [3]. However, the reported effective doses of coronary CT angiography using retrospective ECG gating are rather high, ranging from about 15 to $20 \mathrm{mSv}[4,5]$. Prospective ECG gating with acquisition of axial datasets (also called "step-and-shoot") is an established CT scanning technique that has been used for a long time for coronary artery calcium scoring and has recently also

Editorial Comment on the article of DeFrance et al. (doi: 10.1007/s10554-009-9522-6).

\section{Dewey}

Department of Radiology, Charité, Medical School, Freie Universität, Humboldt-Universität zu Berlin, Berlin, Germany

\section{Dewey ( $\square)$}

Charité-Universitätsmedizin Berlin, Institut für Radiologie, Charitéplatz 1, 10117 Berlin, Germany

e-mail: marc.dewey@charite.de been employed as a very promising concept to reduce the effective dose of coronary CT angiography [6-8].

DeFrance et al. propose prospectively ECG-gated helical (spiral) acquisitions as a very promising new prospective acquisition technique for coronary CT angiography [9]. After helical data acquisition, reconstruction provides axial datasets as for standard retrospective helical acquisitions. The authors show that prospectively ECG-gated helical acquisition has a sufficient image quality while greatly reducing effective dose (mean of $6.9 \mathrm{mSv}$, range: 2.9$10.7 \mathrm{mSv}$ ) compared with standard retrospectively ECG-gated helical acquisition (mean of $16.9 \mathrm{mSv}$, range: $10.1-25.7 \mathrm{mSv}$ ). This is achieved by avoiding exposure-intensive overscanning and increasing the pitch (from about 0.2 to 0.27 ) using prospective helical acquisition. However, their study lacks an important methodological feature since it would have been even more interesting to compare axial stepand-shoot acquisition and prospectively ECG-gated helical acquisition. Such a comparison is not available and would be most interesting in patients with low heart rates. A significant bias is also introduced into this study by comparing two different patient populations: patients with higher heart rates were examined using retrospectively ECG-gated helical acquisition whereas the new prospectively ECGgated technique was used in patients with low heart rates $(<65$ beats per minute). Of the 29 patients examined in the study using retrospective ECGgating, only 10 had heart rates below 65 beats per 
minute (those in whom functional evaluation was required). Thus, this selection bias in the two groups is not negligible and further studies are required to establish the very interesting concept of prospective helical acquisition for coronary CT angiography.

The main advantage of prospectively ECG-gated helical acquisitions is the possibility to switch to standard retrospective ECG-gating if any type of arrhythmia occurs. This is different from prospectively ECG-gated axial acquisition, where arrhythmia rejection works as follows: scanning is stopped if an arrhythmia occurs and is continued in the next beat. However, in case of frequent arrhythmias, this might take pretty long and most of the contrast agent may have left the coronary arteries before scanning has been completed. Thus, prospectively ECG-gated helical acquisition appears to have an advantage in this regard. But, this advantage is also a disadvantage in terms of effective dose, which will of course increase to a greater extent when switching to standard retrospective ECG-gated helical acquisition becomes necessary. In their study population of 57 patients in whom prospective acquisition was performed, DeFrance and coworkers noted this switch to standard retrospective ECG-gated helical acquisition in only two patients with arrhythmia or a heart rate of over 65 beats per minute. In those patients, however, the effective dose increased to about 20-21 mSv. That is why a comparative study with the axial stepand-shoot scanning is of great interest and the scientific community is encouraged to fill this gap.

The drawback of all prospective acquisition approaches that involve more than one heart beat, including the prospective spiral mode reported by DeFrance et al., is of course the susceptibility to ECG-related artifacts and, which is even more important in heavy patients, table-transition-related artifacts [10]. These artifacts can only be eliminated by avoiding the step portion in the "step-and-shoot" techniques and can be accomplished by imaging in a single heartbeat as recently suggested using either 320-row coronary CT angiography $[11,12]$ or highpitch prospective spiral acquisition [13, 14]. However, the scanners required for these two approaches (320-row CT and fast dual-source 64-row CT) are not as widely available as standard 64-row CT scanners. Thus, the concept put forward by DeFrance et al. in the current issue of the Journal is of great interest to the cardiovascular imaging community.

\section{References}

1. Hamon M, Biondi-Zoccai GG, Malagutti P, Agostoni P, Morello R, Valgimigli M (2006) Diagnostic performance of multislice spiral computed tomography of coronary arteries as compared with conventional invasive coronary angiography: a meta-analysis. J Am Coll Cardiol 48:1896-1910

2. Vanhoenacker PK, Heijenbrok-Kal MH, Van Heste R et al (2007) Diagnostic performance of multidetector CT angiography for assessment of coronary artery disease: metaanalysis. Radiology 244:419-428

3. Dewey M (2008) Chapter 5. Indications. In: Dewey M (ed) Coronary CT angiography. Springer, Heidelberg

4. Hausleiter J, Meyer T, Hermann F et al (2009) Estimated radiation dose associated with cardiac $\mathrm{CT}$ angiography. Jama 301:500-507

5. Raff GL, Chinnaiyan KM, Share DA et al (2009) Radiation dose from cardiac computed tomography before and after implementation of radiation dose-reduction techniques. Jama 301:2340-2348

6. Earls JP, Berman EL, Urban BA et al (2008) Prospectively gated transverse coronary CT angiography versus retrospectively gated helical technique: improved image quality and reduced radiation dose. Radiology 246:742-753

7. Gopal A, Mao SS, Karlsberg D et al (2009) Radiation reduction with prospective ECG-triggering acquisition using 64-multidetector computed tomographic angiography. Int J Cardiovasc Imaging 25:405-416

8. Isma'eel H, Hamirani YS, Mehrinfar R et al (2009) Optimal phase for coronary interpretations and correlation of ejection fraction using late-diastole and end-diastole imaging in cardiac computed tomography angiography: implications for prospective triggering. Int $\mathrm{J}$ Cardiovasc Imaging 25:739-749

9. DeFrance T, Dubois E, Gebow D, Ramirez A, Wolf F, Feuchtner GM (2009) Helical prospective ECG-gating in cardiac computed tomography: radiation dose and image quality. Int J Cardiovasc Imaging. doi:10.1007/s10554009-9522-6

10. Husmann L, Herzog BA, Burkhard N et al (2009) Body physique and heart rate variability determine the occurrence of stair-step artefacts in 64-slice CT coronary angiography with prospective ECG-triggering. Eur Radiol

11. Rybicki FJ, Otero HJ, Steigner ML et al (2008) Initial evaluation of coronary images from 320-detector row computed tomography. Int J Cardiovasc Imaging 24:535-546

12. Dewey M, Zimmermann E, Deissenrieder F et al (2009) Noninvasive coronary angiography by 320 -Row CT with lower radiation exposure and maintained diagnostic accuracy: comparison of results with cardiac catheterization in a headto-head pilot investigation. Circulation 120:867-875

13. Lell M, Marwan M, Schepis T et al (2009) Prospectively ECG-triggered high-pitch spiral acquisition for coronary CT angiography using dual source CT: technique and initial experience. Eur Radiol 19:2576-2583

14. Ertel D, Lell MM, Harig F, Flohr T, Schmidt B, Kalender WA (2009) Cardiac spiral dual-source CT with high pitch: a feasibility study. Eur Radiol 19:2357-2362 